\title{
Glossaire DOHaD
}

Anthropique: du grec anthropos (homme). Relatif à l'activité humaine. Qualifie tout élément provoqué directement ou indirectement par l'action de l'homme : érosion des sols, pollution par les pesticides des sols, relief des digues, etc.

Charge allostatique : le stress et les différences individuelles dans la perception des évènements externes (coping) créent des variations du niveau d'activité du système nerveux central (axe hypothalamo-hypophysaire), du système nerveux autonome, du système neuroendocrine (axe hypothalamo-corticosurrénalien) et des processus inflammatoires. Sur une longue période, cette dynamique neuroendocrine peut altérer des mécanismes physiologiques favorisant le développement d'états pathologiques. La charge allostatique renvoie à cette idée d'usure biologique découlant de l'adaptation à un environnement délétère : « La contrainte physique sur le corps, produite par les hauts et les bas répétés des systèmes physiologiques, les changements dans le métabolisme et l'impact de l'usure sur un certain nombre d'organes et tissus, peut prédisposer l'organisme à la maladie. Nous définissons cet état de l'organisme comme la charge allostatique » [1].

\section{Conditionnement et/ou programmation : voir Programmation et/ou Conditionnement}

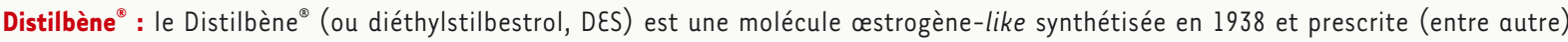
dans la prévention de l'accouchement prématuré. Ce médicament a été largement prescrit comme préventif des fausses-couches dans les années 1960-1970 aux États-Unis et en Europe. Il a été retiré du marché suite aux observations d'anomalies génitales gravissimes sur les enfants des femmes traitées. Des malformations génitales ont été décrites chez les enfants (des deux sexes) issus de ces grossesses, ainsi que des cancers du vagin chez les petites filles. Il a été aussi rapporté qu'à la $3^{e}$ génération, il persiste un risque accru de malformations des organes génitaux externes, notamment des garçons dont les grand-mères avaient été traitées par le DES (par exemple: des anomalies génito-urinaires avec une hypospade chez les garçons de femmes ayant été exposées au DES in utero). L'utérus distilbène : le distilbène induit des malformations de l'utérus ainsi qu'une forme rare de cancer de l'utérus, l'adénocarcinome à cellules claires.

Empreinte parentale : mécanisme essentiel du développement des mammifères, par lequel certains locus (plus d'une centaine) sont réprimés pour un des deux allèles, en fonction de son origine parentale (père ou mère). Les gènes soumis à empreinte parentale sont différents des autres gènes, puisqu'un seul des deux allèles est exprimé dans les cellules somatiques. Des mécanismes épigénétiques particuliers sont associés à cette expression monoallélique des gènes soumis à empreinte, qui peut varier dans le temps et selon les tissus.

Épimutation : modification d'une ou de plusieurs marques épigénétiques stables au cours des divisions cellulaires et entraînant, à l'instar d'une mutation dans la séquence de l'ADN, une modification de l'expression d'un ou de plusieurs gène(s). La grande majorité des études d'épimutations ont porté sur des modifications de la méthylation de l'ADN.

Génome et épigénome : le génome comprend 3 milliards des 4 types de nucléotides A T C G qui constituent notre ADN, dont environ $2 \%$ codent pour nos 23000 gènes. Chaque individu reçoit de chacun de ses parents un génome haploïde qui constitue son patrimoine génétique. Notre patrimoine génétique, hérité de nos parents, est lu grâce au « code génétique » qui permet de traduire la séquence des gènes en protéines, un codon étant formé de 3 bases correspondant à un acide aminé de la protéine. L'épigénome est lui constitué de l'ensemble des marques épigénétiques disposées sur l'ADN (méthylation) et sur les histones (modifications post-traductionnelles des histones ou MPT). Chaque type cellulaire dispose de son épigénome propre qui dépend des marques apposées, ou enlevées, par un ensemble d’enzymes correspondant à la «machinerie épigénétique » sous l'influence de l'environnement, du sexe, de l'âge, et des conditions physiopathologiques. Un « code de la méthylation » et un « code des histones » permettent d'établir, d'effacer, de reconnaître les différents types de combinatoires de marques pour les traduire en expression des gènes.

Hormono-mimétisme : I’hormono-mimétisme définit la propriété de certaines molécules exogènes qui ne sont pas des hormones, au sens où elles ne sont pas produites par une glande endocrine, mais qui peuvent dans certaines conditions exercer des activités reproduisant, tout ou en partie, l'action de l'hormone qu'elles miment. Dans le contexte des perturbateurs endocriniens, il a été montré que plusieurs molécules anthropiques (d'origine industrielle) ou naturelles (d’origine végétale) pouvaient exercer des activités œstrogénomimétiques parce qu'elles «mimaient » l'action des œstrogènes dans une situation donnée.

Marques épigénétiques : les marques épigénétiques désignent les différentes formes de méthylation de l’ADN et des modifications posttraductionnelles des histones (il en existe une centaine). Les marques épigénétiques correspondent à diverses petites molécules, des groupements méthyl, acétyl, phosphoryl, etc., que les enzymes de la « machinerie épigénétique » fixent et enlèvent sur l'ADN et les histones, ces protéines autour desquelles l'ADN s'enroule et qui forment les nucléosomes. Mais, si elles sont associées à l'activation ou à l'extinction de certains gènes, ces marques n'en sont pas nécessairement la cause. Parmi les protéines de la machinerie épigénétique, il existe aussi d'indispensables auxiliaires, des protéines qui se fixent sur l'ADN méthylé ou les modifications post-traductionnelles des histones et lisent leur «code», des agents « recruteurs », des chaperons et des co-chaperons, etc. Les facteurs de régulation comme les facteurs de transcription (FT) ont un rôle clé. Enfin les structures du cytosquelette et de la matrice extracellulaire peuvent également représenter des 
déterminants de la forme de la cellule, de sa fonction et de sa destinée. Mais il est encore difficile de réconcilier les données connues pour parvenir à un scénario fédérateur.

Néomutation : mutation se produisant de novo, soit dans les gamètes parentaux, soit très tôt après la fécondation, et présente dans la quasi totalité des cellules d'un individu. Si cette mutation se produit un peu plus tardivement, l'individu sera une mosaïque, ou bien encore la mutation sera uniquement somatique et sera limitée à un type cellulaire comme cela est observé pour une mutation tumorale à l'origine du développement d'un cancer.

Nomenclature des marques épigénétiques : il existe une nomenclature pour les marques des histones qui mentionne tout d’abord le numéro de l'histone (ou du variant), puis la position de l'acide aminé modifié, puis le type de modification. Ainsi me traduit une méthylation, ad, une acétylation, etc. Puis le nombre de groupements correspondants : une trimethylation de l'histone H3 (nom de l'histone) sur la lysine (K) 4 sera H3K4me3 [2].

Périnatal : correspond, chez l'homme, à la période entre la $28^{\mathrm{e}}$ semaine d'aménorrhée et le $8^{\mathrm{e}}$ jour suivant la naissance. Mais on emploie aussi largement le terme «périnatal » dans les modèles animaux. Dans ce cas, la période couvre généralement toute la gestation jusqu’au sevrage.

Plasticité développementale, plasticité adaptative : capacité de l'épigénome, lors du processus développemental, à se modifier pour donner, à partir de cellules totipotentes, les différents types cellulaires tout en s'adaptant en réponse aux conditions de l'environnement qui peuvent ainsi changer le paysage épigénétique.

Programmation ou conditionnement : utilisé initialement pour désigner les changements au cours du développement fœtal (programmation fœtale), il s'est progressivement étendu à toute période du développement (programmation développementale). II repose sur les changements morphologiques et fonctionnels des cellules et tissus somatiques et extraembryonnaires induits par l'exposition précoce à des facteurs environnementaux. Contrairement aux marques épigénétiques elles-mêmes, qui sont par nature flexibles et réversibles, certaines de ces modifications fonctionnelles des tissus ne sont plus réversibles. La société française DOHaD propose de remplacer dans certains contextes le terme programmation par le terme conditionnement qui exprime mieux les faits observés que le terme programmation trop déterministe. En effet, le plus souvent ce conditionnement n'affecte pas la santé d'un sujet dès la naissance, mais affectera sa capacité de réponse à des environnements ou style de vie délétères, qu'il est donc possible d'éviter. D’où la notion des deux événements nécessaires à l'apparition d'une maladie, avec des facteurs environnementaux qui, dans un premier temps « conditionnent » l'individu, puis d'autres influences environnementales qui, dans un second temps, permettront de « révéler » ce conditionnement et aboutiront à l'apparition d'une maladie.

Reprogrammation épigénétique : ce terme, différent du terme programmation, désigne l'ensemble des phases d'effacement puis d'établissement de nouvelles marques épigénétiques. Il existe deux grandes phases de reprogrammation. La première, après la fécondation, permet le passage du zygote - constitué des génomes haploïdes parentaux caractérisés par les épigénomes caractéristiques des gamètes maternels et paternels - à des cellules souches totipotentes; la seconde, dans les cellules germinales primordiales, permet la reprogrammation de cellules germinales spécifiques du sexe de l'individu. Bien que l'effacement des marques acquises par les parents soit quasi complet, certaines séquences semblent pouvoir échapper à cet effacement, à des stades qui peuvent être différents selon que les épigénomes soient d'origine maternelle ou paternelle. Cet échappement à l'effacement, pour de rares séquences, pourrait permettre la transmission transgénérationnelle de certains caractères acquis.

Retard de croissance intra-utérin (RCIU) : il correspond à une altération de la courbe de croissance intra-utérine qui se traduit par un poids trop faible pour l'âge gestationnel (ou le terme) de la grossesse en comparaison à une courbe de référence. On utilise le plus souvent des abaques présentant les percentiles, mieux adaptés à la description de répartitions irrégulières; deux écarts types ( \pm 2 DS) contiennent $95 \%$ d'une population, soit du $2,5^{\mathrm{e}}$ percentile au $97,5^{\mathrm{e}}$ percentile. La normalité se situe entre le $10^{\mathrm{e}}$ et le $90^{\mathrm{e}}$ percentile, la majorité d'individus se situant autour de la médiane, au $50^{\mathrm{e}}$ percentile. Les nouveau-nés situés en dessous du $10^{\mathrm{e}}$ percentile sont considérés comme ayant un trop faible poids, ce qui correspond à $10 \%$ des naissances.

Systèmes Polycomb/Trithorax : ces complexes de protéines interviennent dans le maintien de l'expression des gènes. Certaines de ces protéines ont des activités enzymatiques modifiant les marques des histones. Parmi celles-ci, les complexes Polycomb (PcG) provoquent la triméthylation de la lysine 27 de l'histone 3 (H3K27me3) - une marque associée à une répression d'expression des gènes -, tandis que les complexes du groupe Trithorax ( $\operatorname{rr} \mathrm{KG}$ ) induisent une triméthylation de la lysine 4 de l'histone 3 (H3K4me3), marque associée à une chromatine transcriptionnellement active.

Théorie de l'attachement : théorie fondée par le psychiatre et psychanalyste anglais John Bowlby après la Seconde Guerre mondiale. Cette théorie souligne que l'enfant a besoin de développer un lien d'attachement, avec au moins une personne qui prend soin de lui de façon cohérente et continue, pour que son développement social et émotionnel se réalise normalement, et que ses liens futurs se construisent de manière adaptée.

Transgénérationnel, inter- ou multigénérationnel : les termes transgénérationnel d'une part, et intergénérationnel ou multigénérationnel d'autre part, sont utilisés pour rendre compte de voies spécifiques (somatique ou par la lignée germinale via les gamètes) et du nombre de générations de transmission. Le terme intergénérationnel se rapporte le plus souvent à des transmissions via la mère et tient compte 
d'effets ou de réponses observés sur la progéniture, de troubles métaboliques présents chez la mère (obésité, diabète, etc.) mais aussi d'impacts de l'environnement ayant pu toucher, au niveau épigénétique, les ovocytes de la mère. À moins de faire un transfert d'embryon, il n’est pas possible de départager les effets via la lignée germinale de ceux acquis via la lignée somatique. II faut ainsi attendre la génération F3, qui n'aura pas pu être exposée directement, pour pouvoir invoquer une transmission transgénérationnelle via la lignée germinale et, en cas de transmission paternelle, la génération F2. Les transmissions mutigénérationnelles s'adressent aux situations où l'exposition persiste sur plusieurs générations. Pourtant, on peut utiliser aussi le terme transgénérationnel dans son sens général pour décrire les conséquences mesurables sur une génération (la progéniture) d’une exposition de la mère ou du père (avant la conception) ou de leurs parents ou ancêtres respectifs. Le terme « effets » transgénérationnels communément utilisé, s'adresse en réalité à des « réponses » transgénérationnelles. Ces réponses peuvent correspondre à un cercle vicieux avec propagation des effets initiaux, ou bien à une résilience invoquant un processus d'adaptation. Les réponses des descendants peuvent donc, pour un même impact initial, se manifester très différemment des effets observés lors de l'exposition de la première génération.

\section{RÉFÉRENCES}

1. McEwen BS, Stellar $\varepsilon$. Stress and the individual : mechanisms leading to disease. Arch Intern Med $1993 ; 153: 2093-101$.

2. Allis CD, Berger SL, Cote J, et al. New nomenclature for chromatin-modifying enzymes. Cell 2007 ; $131: 633-6$.

B

ertrand Jordan a participé à l'extraordinaire aventure de la biologie moléculaire, encore balbutiante dans les années 1960, mais qui a complètement révolutionné les sciences du vivant au cours des décennies suivantes.

L'ADN, quasiment inaccessible au début de cette période, intervient maintenant dans d'innombrables recherches, de l'écologie à l'anthropologie, sans oublier bien sûr la médecine dont il fait désormais partie intégrante. Nous pouvons aujourd'hui lire intégralement

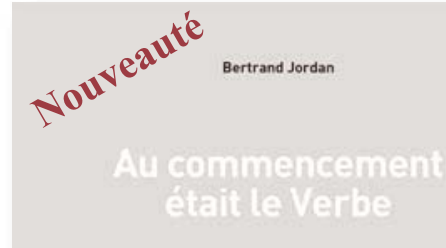

Une histoire personnelle de l'ADN

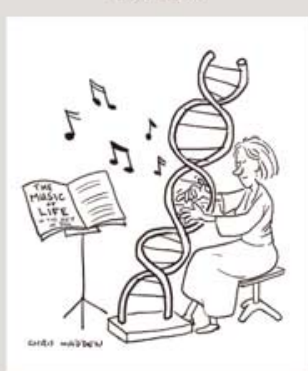

edpsciences

I'ADN d'une personne en quelques heures, et en tirer de précieuses informations pour la prévention et le traitement de nombreuses maladies - et nous n'en sommes qu'au début de cette nouvelle médecine!

Acteur mais aussi témoin de ces avancées, Bertrand Jordan fait ici un récit très personnel et sans langue de bois de sa vie avec l'ADN. Ce livre ne prétend pas être une histoire complète de la biologie moléculaire, mais il illustre son développement, révélant parfois le «dessous des cartes » grâce aux expériences vécues par son auteur.

$3<$

UU À retourner à EDP Sciences, 109, avenue Aristide Briand, 92541 Montrouge Cedex, France

Tél. : 0141177405 - Fax : 0149850345 - $\varepsilon$-mail : edk@edk.fr

NOM :

Adresse :

Code postal

Prénom

Pays :

Fonction

Je souhaite recevoir

Au commencement était le Verbe : $20 €+3 €$ de port $=23 € \pi C$

en .................. exemplaire, soit un total de

Ville :

$\square$ Par chèque, à l'ordre de EDP Sciences

$\square$ Par carte bancaire : $\quad \square$ Visa $\square$ Eurocard/Mastercard

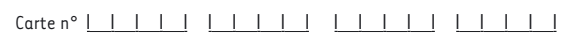

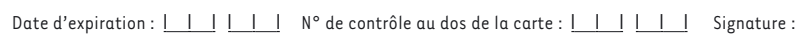

\section{> Grâce à $m / s$, vivez en direct les progrès des sciences biologiques et médicales}

\section{Abonnez-vous}

à médecine/sciences

\section{Bulletin d'abonnement} page 130 dans ce numéro de $\mathrm{m} / \mathrm{s}$

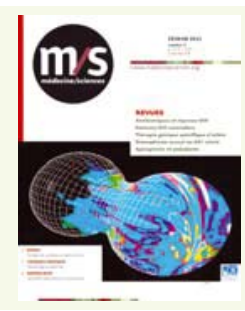

